\title{
Cementless Total Hip Arthroplasty After an Iatrogenic Subtrochanteric Fracture due to Hardware Removal: A Case Report
}

\author{
Francesco Castagnini ${ }^{1 *}$, Giovanni Bracci ${ }^{1}$, Enrico Tassinari ${ }^{1}$, Federico Biondi ${ }^{1}$ \\ 'Ortopedia-traumatologia e Chirurgia Protesica e dei Reimpianti di Anca e Ginocchio, IRCCS Istituto Ortopedico \\ Rizzoli, Bologna, Italy
}

*Corresponding Author: Francesco Castagnini, M.D., Ortopedia-traumatologia e Chirurgia Protesica e dei Reimpianti di Anca e Ginocchio, IRCCS Istituto Ortopedico Rizzoli, Bologna, Italy. Tel: +39-0516366148, Fax: +39-051-

6366416, Email: francescocastagnini@hotmail.it

Received April 26, 2019; Accepted July 15, 2019; Online Published August 26, 2019

\begin{abstract}
Introduction: Total hip arthroplasty (THA) after proximal femoral fixation is a challenging procedure due to possible hardware-related complications.

Case presentation: A 78-year-old female with hip osteoarthritis had a proximal femoral osteotomy fixed using a blade plate in the same femur 41 years ago. A two-step approach was planned. After a challenging hardware removal, an iatrogenic subtrochanteric fracture below the degenerated hip occurred after three months. THA with a tapered long stem was successfully performed with no need for additional osteosynthesis, and good results were seen two years later.

Discussion: THAs in subtrochanteric fractures are technically demanding but feasible in selected cases. Hardware removal before THA implantation may carry important risks, and the surgical team should be prepared to perform arthroplasty in case of complications.

Conclusion: THA in a subtrochanteric fracture below hip osteoarthritis is a feasible option in selected cases.

Keywords: Arthroplasty, Femoral Fracture, Osteotomy, Stress
\end{abstract}

\section{Introduction}

Total hip arthroplasty (THA) after proximal femoral fixation is a challenging procedure. ${ }^{1-5}$ High rates of complications, predominantly periprosthetic fractures, abductor dysfunction, and medical complications, were reported in the largest case series. The clinical results of THAs after femoral fixation were significantly inferior to control groups. ${ }^{1-5}$ Specifically, some hardware-related challenges, such as stress shielding, bone holes, infection, and retained screws, are noteworthy. If hardware removal and THA are performed in the same surgical step, pressurization of the cement can be impaired, fractures can occur due to weak bone and stress concentration, and infections related to previous surgeries cannot be ruled out. $^{1-5}$ Thus, a previous preparatory surgery to remove the hardware and, when needed, to take tissue samples to exclude septic complications may be desirable, possibly making a subsequent THA surgery shorter, safer and less demanding. ${ }^{2}$ In the case of a THA after a previous femoral osteotomy, however, hardware removal was reported as troublesome in $24 \%$ of patients. ${ }^{3}$ Herein, a case of hip osteoarthritis after a proximal femoral osteotomy is presented. The patient was treated with a two-step approach (hardware removal preceding THA). Soon after the hardware removal, an iatrogenic subtrochanteric fracture occurred, which was successfully treated with a primary tapered long-stemmed THA without any additional osteosynthesis.

\section{Case Presentation}

A 78-year-old female was scheduled for THA in a dysplastic left hip. The patient complained about a recent onset of groin pain and a reduction in the articular excursion due to degenerative joint disease. The left hip had been treated with an intertrochanteric osteotomy 41 years prior to this case. A blade plate had been used to fix the osteotomy (Figure 1). The patient showed no local signs of infection, and her C-reactive protein was $0.32 \mathrm{mg} / 100 \mathrm{~mL}$ (normal value: $<0.50 \mathrm{mg} / 100 \mathrm{~mL}$ ). The patient was scheduled for a twostep approach (hardware removal preceding THA). The patient's age and the quality of the bone were considered, and the two-step approach was adopted in order to restore

Copyright $\odot 2019$ The Author(s). This is an open-access article distributed under the terms of the Creative Commons Attribution License (http:// creativecommons.org/licenses/by/4.0), which permits unrestricted use, distribution, and reproduction in any medium, provided the original work is properly cited. 
the bone stock after hardware removal and reduce the risk of iatrogenic fractures during stem positioning.

The patient had a body mass index of $26.4 \mathrm{~kg} / \mathrm{m}^{2}(165$ $\mathrm{cm}$ and $72 \mathrm{~kg}$ ) and unremarkable comorbidities. She was classified as ASA (American Society of Anesthesiologists) grade 2 . The hardware removal was performed using a lateral approach on the previous scar. The removal was difficult; only one screw could be easily removed. The other 4 screws were removed using a trephine; thus, the lateral cortex was weakened. Due to possible additional risks related to the reduced bone stock (intraoperative fractures, stem subsidence), the conversion to a single-stage procedure, that is, immediate THA, was not performed.

The patient was then advised not to weight-bear until a proper bone stock restoration was achieved. After three months, while a toe touch weight-bearing was allowed, the patient started complaining of hip pain. After 15 days, the patient felt pain, was unable to walk, and had a shortened left lower limb $(-2 \mathrm{~cm})$. An iatrogenic subtrochanteric fracture occurred (Figure 1). To treat the fracture below the osteoarthritic hip, a revision THA was implanted. The same lateral incision was used, allowing a direct visualization of the fracture. First, the acetabulum was prepared as usual, and then a trabecular metal cup with a longevity polyethylene liner was implanted (Zimmer, Warsaw, US). On the femoral side, a proper fracture reduction was performed and maintained using reduction forceps. The fracture reduction was directly controlled while the femoral canal was prepared using progressive reamers. A tapered 190-cm long stem (Wagner, Zimmer, Warsaw, US) was implanted in order to bypass the fracture. Due to the good fracture stability, no additional osteosynthesis was performed. The lateral cortical bone loss was filled with autologous bone from reaming. The implant was then reduced using a $28-\mathrm{mm}$ metal head (Figure 2).

After surgery, weight-bearing was not allowed. Toe touch weight bearing was allowed after 45 days. Complete weight bearing was delayed until the third month after surgery (Figure 2).

After three years, the patient was painless, active, and independent, requiring a walking cane for long walks (Harris Hip score: 91 points). Radiographic examination revealed the healing fracture and a well-positioned implant with no signs of stem subsidence (Figure 2).

\section{Discussion}

Previous proximal femoral fixation was associated with a more demanding THA conversion., ${ }^{2,3}$ In particular, the presence of hardware was described as one of the most notable challenges, influencing the surgical approach, blood loss, operative time, and the implant choice, with increased risk of infections, fractures, and possible loosening. ${ }^{2,3,5}$ Despite the need for two surgical procedures, a two-step approach may provide a safer solution: the hardware can be removed in the first step and then, after the bone stock restoration, the THA can be performed. ${ }^{2,5}$ However, hardware removal before THA is associated with high rates of challenges and complications (24\%). Systemic osteoporosis and local bone stock reduction due to stress protection have been reported as predisposing factors for fractures. ${ }^{1,3}$ In this case report, the age of the patient, the hardware-related stress shielding, and the arduous removal associated with broken screws requiring burs and trephines severely weakened the lateral cortex, resulting in a transverse subtrochanteric fracture. The conversion to a single-step procedure may have been beneficial in this case; however, due to the reduced bone stock and no strict indication (like frank fracture) to perform a demanding and risky surgery, the THA implantation was delayed. When the fracture occurred below the osteoarthritic hip, the chosen treatment was a THA with a revision stem. In fact, a simple reduction and fixation would have not

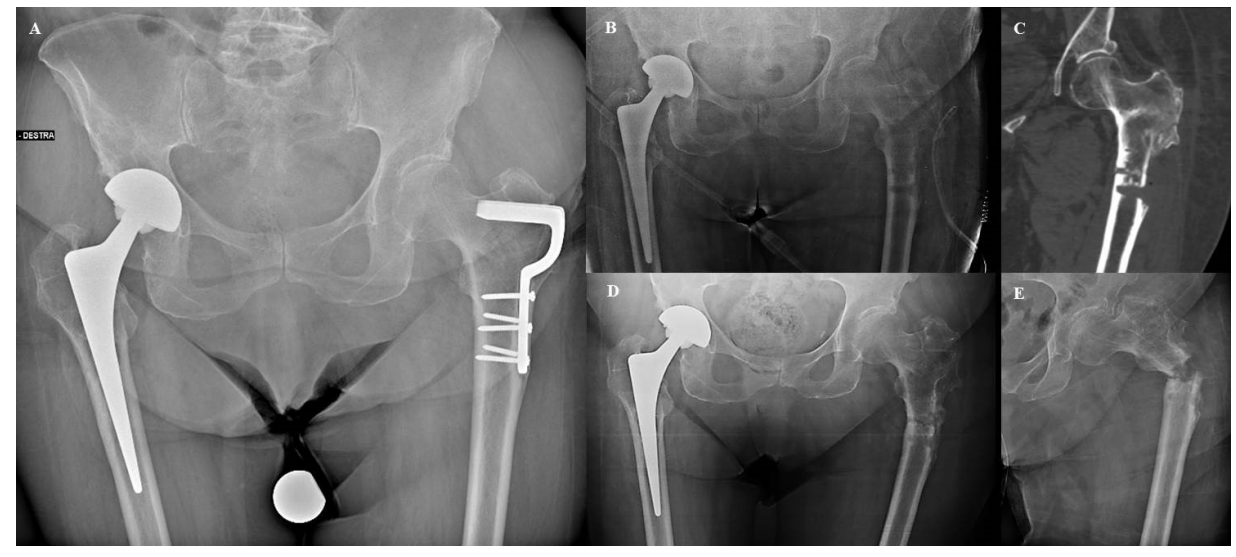

Figure 1. Anterior-posterior X-ray of the pelvis showed an osteoarthritic hip in a mild dysplastic morphology: a femoral varus osteotomy with a blade-plate fixation was performed 41 years before (A). The anterior-posterior X-ray of the pelvis soon after the challenging hardware removal showed severe lateral bone loss and a very thin medial cortex at the level of the removed bone screws (B). The frontal CT scan 15 days later confirmed the situation (C). After 90 days, in two serial anterior-posterior X-rays of the pelvis, a varus angulation was evident, which ended in an iatrogenic, transverse subtrochanteric fracture (D and E). 


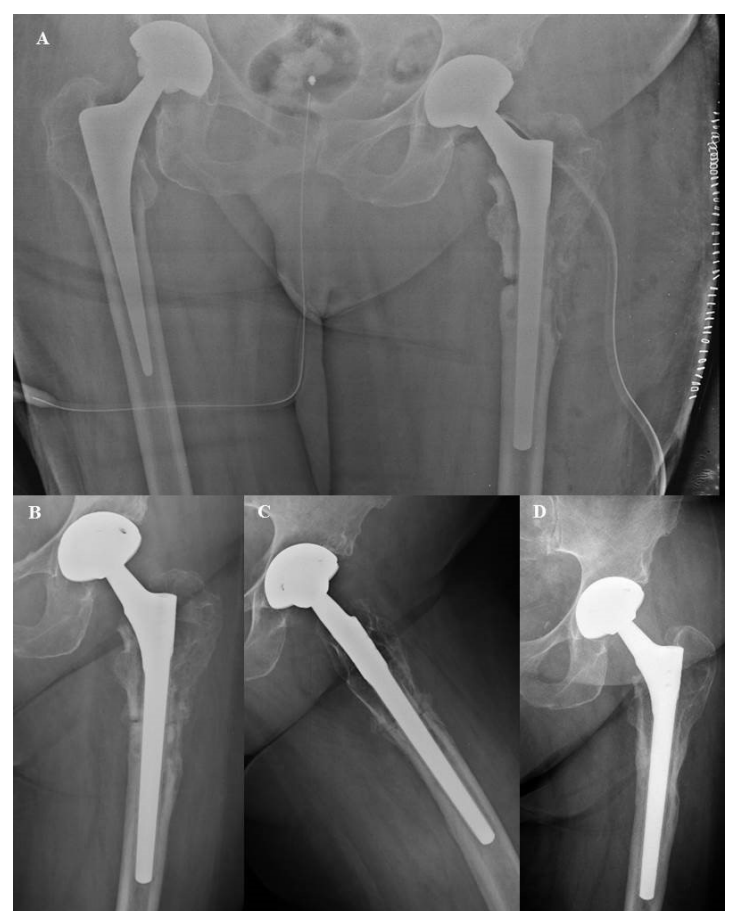

Figure 2. A tapered long stem was implanted, bypassing the fracture and obtaining a good reduction and fixation (A). After 45 days (B and C) and 3 years (D), the fracture showed progressive signs of healing and good osseointegration of the stem.

addressed the articular degeneration and would have required another surgical procedure. Thus, a cementless highly porous cup with a polyethylene insert, a metal ball and a conical long stem was implanted. A conical stem may have provided a good distal (diaphyseal) fixation, bypassing the fracture and achieving a satisfying reduction, but as reduction and fixation was brilliantly achieved by the long stem, no additional osteosynthesis was required.

The use of the cementless long stem in cases of subtrochanteric fracture has been amply reported. Yuasa et al described a single case of atypical subtrochanteric fracture below an arthritic hip after a prolonged bisphosphonate therapy. ${ }^{6} \mathrm{~A}$ tapered modular THA with an additional osteosynthesis was adopted with good results. A 12-patient case series was provided by Oztürkmen et al. ${ }^{7}$ The subtrochanteric fractures below arthritic hips were treated with cannulated, tapered long stems (Helios, Biomet, Warsaw, US). Cerclages and cables were used to avoid fracture propagation. A distal fracture and a greater trochanter fracture occurred. Encouraging results were described, with a mean Harris Hip score of 83 points and only one case of stem subsidence. The authors postulated that such a single procedure was demanding, but in the case of elderly patients, it might have provided a good alternative to two-step surgeries (fixation+THA).

Despite the appropriate differences, THAs after failed subtrochanteric fracture fixation may provide a good comparison with our case report. Only a few patients included in large case series have been described. Enocson et al reported 25 subtrochanteric fractures treated with total or partial hip arthroplasty procedures. Despite the inclusion of different approaches and devices, long stem implants performed better with a reduced risk of revision. ${ }^{8}$ Thakur et al and Weiss et al respectively described 5 failed intertrochanteric fracture fixations with subtrochanteric extension and 20 failed subtrochanteric fracture fixations treated with tapered long stems; their clinical and radiographic results were good., ${ }^{9,10}$

\section{Conclusion}

This case report highlighted two hot topics in complex THAs: the challenges presented by femoral hardware presence and the lack of consensus about the treatment of subtrochanteric fractures below osteoarthritic hips. Femoral hardware should be removed before THA, allowing bone stock restoration and ruling out latent sepsis. Unfortunately, a remote risk of fracture should be taken into account. When a difficult hardware removal is anticipated, a resurfacing or a mini-stem option should be evaluated, weighting the pros (no hardware removal) and the cons (metallosis) of this procedure. In the current case, the conversion to a single-stage procedure (THA with a tapered stem) immediately after the complicated hardware removal may have been the best solution; however, this procedure is really demanding, and the risk of intraoperative fractures and subsequent stem subsidence may be very high, particularly in elderly patients. Thus, this procedure should be limited to very selected cases in which the two-stage procedure is too physically demanding for the patient. In cases of a subtrochanteric fracture below an osteoarthritic hip, when a single procedure is recommended, a cementless tapered long stem implant seems to be a viable option. This stem bypasses the fracture, maintains a proper reduction, and achieves a good diaphyseal fixation. Additional osteosynthesis can be avoided if a good stability of the fracture is achieved with the stem acting as an internal fixator.

\section{Authors' Contributions}

The authors contributed equally to this work, collecting and analyzing data (FC, ET, FB, GB) and preparing the manuscript (FC). The local ethical committee approved the study, and signed consent was obtained from the patient.

\section{Conflict of Interest Disclosures}

The authors declare that they have no conflicts of interest.

\section{Ethical Approval}

The current study met the ethics standards described in the Declaration of Helsinki and Singapore. The institutional review board (IRB) approved the study, and the patient gave informed consent for this case presentation.

\section{References}

1. BarquetA, Giannoudis PV, GelinkA. Femoral neck fractures after removal of hardware in healed trochanteric fractures. Injury. 
2017;48(12):2619-2624. doi:10.1016/j.injury.2017.11.031.

2. Sassoon AA, Trousdale RT. Technical considerations in total hip arthroplasty after femoral and periacetabular osteotomies. Orthop Clin North Am. 2012;43(3):387-393. doi:10.1016/j. ocl.2012.05.006.

3. Ferguson GM, Cabanela ME, Ilstrup DM. Total hip arthroplasty after failed intertrochanteric osteotomy. J Bone Joint Surg Br. 1994;76(2):252-257. doi:10.1302/0301-620X.76B2.8113286.

4. Pui CM, Bostrom MP, Westrich GH, et al. Increased complication rate following conversion total hip arthroplasty after cephalomedullary fixation for intertrochanteric hip fractures: a multi-center study. J Arthroplasty. 2013;28(8 Suppl):45-47. doi:10.1016/j.arth.2013.04.048.

5. Boos N, Krushell R, Ganz R, Muller ME. Total hip arthroplasty after previous proximal femoral osteotomy. J Bone Joint Surg Br. 1997;79(2):247-253. doi:10.1302/0301-620X.79B2.0790247.

6. Yuasa T, Maezawa K, Taniguchi Y, Aritomi K, Kaneko K. Atypical Subtrochanteric Femoral Fracture Below an Arthritic Hip: A Case Report. JBJS Case Connect. 2015;5(1):e1-e6. doi:10.2106/jbjs.cc.n.00030.
7. Oztürkmen $Y$, Uzümcügil $O$, Sükür $E$, Karamehmetoğlu $M$, Caniklioğlu $M$. The use of a cementless modular stem in the treatment of subtrochanteric femoral fractures in conjunction with ipsilateral coxarthrosis. Orthopedics. 2011;34(1):13. doi:10.3928/01477447-20101123-12.

8. Enocson A, Mattisson L, Ottosson C, Lapidus LJ. Hip arthroplasty after failed fixation of trochanteric and subtrochanteric fractures. Acta Orthop. 2012;83(5):493-498. doi:10.3109/174 53674.2012.688724.

9. Weiss RJ, Karrholm J, Hailer NP, Beckman MO, Stark A. Salvage of failed trochanteric and subtrochanteric fractures using a distally fixed, modular, uncemented hip revision stem. Acta Orthop. 2012;83(5):488-492. doi:10.3109/17453674.20 12.733917

10. Thakur RR, Deshmukh AJ, Goyal A, Ranawat AS, Rasquinha VJ, Rodriguez JA. Management of failed trochanteric fracture fixation with cementless modular hip arthroplasty using a distally fixing stem. J Arthroplasty. 2011;26(3):398-403. doi:10.1016/j.arth.2010.01.103. 